\title{
Anti-ghrelin antibodies in appetite suppression: recent advances in obesity pharmacotherapy
}

This article was published in the following Dove Press journal:

ImmunoTargets and Therapy

9 July 2015

Number of times this article has been viewed

\author{
Velimir Altabas \\ Vanja Zjačić-Rotkvić \\ Department of Endocrinology, \\ Diabetes and Metabolic Diseases, \\ "Mladen Sekso", Clinic for Internal \\ Medicine, University Hospital Center \\ "Sestre milosrdnice", Zagreb, Croatia
}

Correspondence: Velimir Altabas Department of Endocrinology, Diabetes and Metabolic Diseases "Mladen Sekso", Clinic for Internal Medicine, University Hospital Center "Sestre milosrdnice", Vinogradska cesta 29, I 000 Zagreb, Croatia

Tel +3859874I 218

Fax +385 I 3787692

Email velimir.altabas@gmail.com
Abstract: Obesity is a medical condition caused by accumulated excess body fat with negative impact on patients' health, including decreased life expectancy. It has become a major health problem in most developed and developing countries, since the worldwide prevalence of obesity nearly doubled during the last 30 years. Consequently, novel treatments focusing on obesity are being investigated. Potential targets include several pathophysiological mechanisms involved in appetite control affecting multiple organ systems, like adipose tissue; some cell types in the stomach and gut; pancreas; thyroid gland; several hypothalamic areas; and centers located in the brainstem. One of the most important orexigenic neuropeptides is ghrelin, which is produced and secreted primarily by ghrelin cells located in the stomach and duodenum. In humans, plasma ghrelin levels rise when the stomach is empty and fall shortly after meal ingestion. In fat tissue, ghrelin increases fat storage. In the brain, it exerts its orexigenic action through activation of NPY/AgRP neurons in the arcuate nucleus. From the pharmacological point of view, it seems that opposing ghrelin activity could be used as a therapeutic principle in treating obesity. The principal idea of antiobesity drugs is to augment anorexigenic and lipolytic signaling, or to block orexigenic and lipogenic mediators. Recent studies have shown that therapeutic vaccines could be a new approach in the development of antiobesity medications. A vaccine should provoke an immune response to a specific causal factor for a particular disease. Several types of anti-ghrelin vaccines have been developed so far, with significant immune response in terms of rising anti-ghrelin antibodies. However, in the only clinical trial performed yet, the results were disappointing, showing no additional weight loss in the study group. Until now, several studies have demonstrated the "proof of concept", but more studies are required to develop prophylactic and therapeutic vaccines to prevent and/or cure obesity.

Keywords: obesity vaccine, obesity pathophysiology, experimental obesity treatment

\section{Obesity}

Obesity is a medical condition caused by accumulated excess body fat with negative impact on a person's health, including decreased life expectancy. The genetic background for obesity is partly regarded as an evolutionary issue. It has been supposed that development of a thrifty genotype was necessary for human survival in an environment with scarce food supply. Genes that helped to accumulate fat reserves for use as energy during times with less food supply were selected. However, civilization changes starting from the agricultural revolution have converted this evolutionary benefit into a serious metabolic problem resulting in obesity. ${ }^{1-5}$

Throughout almost the entirety of recorded human history, obesity commonly affected only the elite with easier access to food, and it was rather seen as a condition 
associated with wealth and higher socioeconomic status. However, in the modern ages with increased food production, a worldwide epidemic of obesity developed related to increased dietary energy supply and intake, and worsened lifestyle with less physical activity. ${ }^{2}$

The worldwide prevalence of overweight and obesity nearly doubled during the last 30 years, and it currently affects approximately two-thirds of the general population in North America and in Great Britain. ${ }^{6,7}$ Obesity has become a major health problem in most developed and developing countries, since it is associated with increased prevalence of comorbidities like type 2 diabetes mellitus, arterial hypertension, dyslipidemia, and other features of the metabolic syndrome leading to cardiovascular disease, and certain types of cancer. It is also associated with increases in premature mortality, impaired quality of life, and large health care cost, and it presents a real burden for the modern human society. ${ }^{8}$

Over 3.4 million people die each year due to the consequences of being overweight or obese according to data from the World Health Organization. Even more dramatic are data about obesity-related disability showing globally 35.8 million disability adjusted life years, a measure for lost years of healthy life. Of special concern is childhood obesity. ${ }^{8}$ On the contrary, obesity is preventable and weight loss may improve several comorbid conditions associated with obesity. ${ }^{8}$ Consequently, novel treatments focusing on obesity are being investigated.

\section{Energy homeostasis and appetite control}

The amount of body fat in humans seems to be precisely regulated in the overall process of energy homeostasis. Energy homeostasis includes energy intake (the amount and type of consumed food), energy expenditure (the combination of energy used to maintain daily bodily functions plus energy expended through activity and exercise), and energy stores (the size of fat mass). ${ }^{9}$

Pathophysiological mechanisms involved in appetite control affect multiple organ systems. Adipose tissue; some cell types in the stomach and gut; pancreas; thyroid gland; several hypothalamic areas; and centers located in the brainstem have been recognized as tissues and organs directly involved in appetite control. A number of local acting and circulating factors of different origin are identified to modulate food intake and energy balance. In addition, circulating carbohydrates, lipids, and amino acids also affect appetite regulation. ${ }^{10-12}$

Fat tissue consisting of over 1 billion cells constitutes a large endocrine organ that constantly communicates with other tissues by adipocyte-released secretagogues. The most important for weight control among these are leptin and adiponectin. Leptin is secreted almost exclusively from adipose tissue, when the amount of stored fat reaches a certain level. It activates leptin receptors in the arcuate nucleus (ARC) of the hypothalamus, and acts as a satiety signal. On the contrary, weight loss causes a marked decrease in leptin levels which in turn increases appetite. Sustained high leptin concentrations seen in obese people may lead to leptin resistance, making the leptin receptor inactive and decreasing the impact of leptin action. ${ }^{13,14}$

Another adipokine produced exclusively by adipocytes is adiponectin. Adiponectin has a prominent role in decreasing insulin resistance and improving survival of pancreatic $\beta$-cells, enhancing their function. It acts also in the brain mediating weight loss. Its concentration in obese people is decreased. ${ }^{14}$

Enteroendocrine cells located in the stomach and gut wall are another important source of hunger and satiety peptides secreted in response to digestion and absorption of meals, and are able to modulate the endocrine response to food intake. These peptides include ghrelin, cholecystokinin (CCK), glucagon-like peptide 1 (GLP-1), oxyntomodulin (OXM), peptide YY (PYY), pancreatic polypeptide (PP), and amylin. Neuroendocrine signals from the stomach and the more distal part of the gastrointestinal system are sent to the hindbrain, providing information about the meal size, food taste, and chemical content. ${ }^{9}$

Ghrelin is one of the most important orexigenic neuropeptides and is produced and secreted primarily by ghrelin cells located in the stomach and duodenum. Ghrelin cells are also found in the jejunum, lungs, pancreatic islets, gonads, adrenal cortex, placenta, and kidneys. In humans, plasma ghrelin levels rise when the stomach is empty and fall shortly after meal ingestion. Interestingly, after disruption of vagal signaling, ghrelin administration fails to stimulate food intake. Ghrelin receptors are found in the same brain cells as the leptin receptors which promote satiety; they exerts their orexigenic action through activation of NPY/AgRP neurons in the hypothalamic ARC. In fat tissue, ghrelin increases fat storage.

Beyond regulating hunger, ghrelin plays a significant role in regulating the rate of energy use. Ghrelin level raises with weight loss as part of the compensatory response to energy deficit. As a result, humans with increased ghrelin levels feel more hungry and burn fat more slowly. Furthermore, obese patients show reduced circulating ghrelin levels and anorexic patients display increased circulating levels of ghrelin. 
Ghrelin exerts its orexigenic effect through its action on the hypothalamic appetite-regulating pathways by activating NPY/AgRP neurons in the ARC, while in the periphery, ghrelin increases adipose tissue accumulation. Ghrelin is also involved in the activation of the mesolimbic dopaminergic pathways responsible for rewards such as food or alcohol intake. ${ }^{15-17}$

Another neuropeptide, CCK, acts like a satiety promotor. It is synthesized by I-cells and secreted in the duodenum and the proximal part of the small intestine, and causes the release of pancreatic digestive enzymes and bile. As a neuropeptide, CCK mediates satiety by activating CCK receptors located widely throughout the central nervous system. In humans, it has been suggested that CCK administration causes nausea and anxiety, decreases the rate of gastric emptying, and induces a satiating effect. ${ }^{18,19}$

GLP-1 is an incretin, a gut hormone that increases pancreatic insulin secretion. The major source of GLP-1 are the enteroendocrine L-cells in the ileum and proximal colon, which secrete GLP-1 in response to meal ingestion. The GLP-1 receptor is expressed in the heart, kidneys, lungs, pancreas, central nervous system, and peripheral nervous system, including the nucleus tractus solitarius of the dorsal vagal complex and the ARC. GLP-1 action leads to increased insulin secretion in a glucose-dependent manner and decreased glucagon secretion from the pancreas. It increases insulin sensitivity. In the stomach, GLP-1 inhibits acid secretion and gastric emptying, and it decreases food intake by increasing satiety signals in the brain. Administration of GLP-1 induces satiety and weight loss in animals and humans. ${ }^{19,20}$

OXM is another product of the gut L-cells. It is released together with GLP-1 in proportion to energy intake. OXM acts as an anorectic agent through GLP-1 receptor activation in the brain. ${ }^{21}$

The gut hormone PYY is similar in structure to PP and neuropeptide Y (NPY). These peptides mediate their effects through the NPY receptors. The L-cells of the terminal ileum and colon are the major source of PYY. The peak PYY level is related to the calories ingested, suggesting that PYY may signal food ingestion from the gut to appetite-regulating circuits within the brain, but in contrast to NPY, peptide PYY is potently anorexigenic. PYY also stimulates gastrointestinal absorption of fluids and electrolytes, reduces gastric and pancreatic secretions, and delays gastric emptying. ${ }^{19,22}$

PP is released from the pancreas in response to food ingestion. It influences food intake, energy metabolism, and the expression of hypothalamic peptides and gastric ghrelin. Systemic PP administration reduces food consumption in humans, but when PP was administered directly into the brain, it increased food intake. ${ }^{19}$

Amylin, or islet amyloid polypeptide, is co-secreted with insulin from pancreatic $\beta$-cells. Plasma levels of amylin in fasting states are low and increase in response to food ingestion. It plays a role in glycemic regulation leading to slower gastric emptying and promoting satiety, thereby blunting post-prandial spikes in blood glucose levels. The effects of amylin on appetite and gastric emptying are attenuated by vagotomy, suggesting that vagal signaling is important in mediating appetite suppression. ${ }^{23}$

Other important hormones included in energy homeostasis are insulin and thyroid hormones. Insulin enters the brain through capillary endothelial cells via insulin receptorfacilitated transport. The process is insulin-specific and saturable. Insulin receptors are expressed in the mediobasal hypothalamus, especially the ARC, which also expresses the most active form of the leptin receptor. This region is thought to mediate most of insulin's action in the brain, where it acts as a satiety signal, reducing food intake and body weight, which is in contrast to its expected effect, since insulin overdosing may cause hypoglycemia and defensive eating. However, when sufficient glucose to avoid hypoglycemia is present, insulin reduces food intake.

The insulin signal in the ARC also leads to reduced hepatic glucose production via vagal innervation, while simultaneously sending a message to the brain to eat less food. ${ }^{12,24,25}$ Considering the impact of thyroid hormones on appetite control, it is well established that the hypothalamicpituitary-thyroid axis contributes to body weight regulation. Thyroid hormones are known to affect the metabolic rate, appetite, thermogenesis, and body weight.

In the hypothalamus, thyroid hormones increase NPY mRNA leading to increased food intake; and on the contrary, hyperphagia induced by thyroid hormones could be blunted by intracerebroventricular administration of a NPY Y1 receptor antagonist, suggesting that thyroid hormones may increase appetite via NPY. Thyroid hormone administration was also reported to reduce hypothalamic proopiomelanocortin (POMC) expression. ${ }^{11,26}$ In addition, several nutrients have been shown to reduce obesity like L-glutamate and polyunsaturated fatty acids. Fructose had a deleterious effect on weight control. ${ }^{27}$

The brain plays key role in energy homeostasis and appetite control. Overall information including meal content is integrated in the brain where it activates several responses. Appetite control centers are placed in the hypothalamic region that is subdivided into a number of interconnecting 
nuclei: the dorsomedial nucleus, paraventricular nuclei, ventromedial nucleus, and ARC. The ARC is surrounded by a permeable membrane outside the blood-brain barrier, and is therefore reachable for circulating hormones and adipokines from blood.

Besides direct signaling, the ARC is able to receive satiety signals from the dorsal vagal complex situated in the brainstem and from the mesolimbic system.

There are several integrative pathways in the brain relying on some hormone and neuropeptide precursors, neuropeptides, and biogenic amines. Neuropeptides are protein-like substances important in the interaction between neurons, but are smaller than neurotransmitters. Neuropeptides relevant for energy balance include: NPY, Agouti-related protein (AgRP), and cocaine- and amphetamine-regulated transcript (CART), while POMC is a hormone and neuropeptide precursor. POMC may be cleaved into melanocyte-stimulating hormone, adrenocorticotropic hormone, $\beta$-endorphin, and encephalin. Biogenic amines include serotonin, norepinephrine, and dopamine, and regulate appetite, pituitary neuroendocrine function, behavior, and exercise.

Two different neuronal subtypes participate in appetite regulation in $\mathrm{ARC}$, expressing different neuropeptides. One subtype expresses POMC and CART, and acts as an appetite suppressor, while the other one expresses NPY and AgRP and stimulates appetite. Energy homeostasis itself may depend on the balance between these neuronal signals.

POMC/CART and NPY/AgRP in ARC act like first order neurons, in which signals are forwarded to secondorder neurons in other hypothalamic nuclei (ventromedial nucleus, dorsomedial nucleus, paraventricular nuclei, lateral hypothalamic area). Second-order neurons terminate in special areas in the caudal brainstem, limbic system, and brain cortex, involving them in the integration of satiety signals and feeding control..$^{9,25,27}$

\section{Obesity treatment}

Today, in contrast to the complexity of appetite regulation and energy balance, there are only a few treatment options to alleviate or even cure obesity. These options include lifestyle changes, medical treatment, and bariatric surgery. The goal of lifestyle modification is to change eating habits to lower energy intake, to increase physical activity, and to increase energy expenditure. When successful, both are associated with clinically meaningful improvements in several cardiovascular disease risk factors, including prevention of type 2 diabetes. ${ }^{8,28}$ Lifestyle changes seem to be the easiest way to treat obesity, but in matter of fact it is a time- and effort-consuming way to manage obesity. They are related to a large proportion of failure, weight regain, and finally, loss of motivation. ${ }^{29,30}$

Knowledge regarding appetite regulation has vastly expanded in recent years providing targets for further antiobesity drug development. To date, five weight loss agents have been approved by the US Food and Drug Administration (FDA): orlistat, naltrexone/bupropion, phentermine/ topiramate, lorcaserin, and liraglutide. These medicines may be divided into several different types of drugs. Orlistat is an inhibitor of fat absorption, acting as a lipase inhibitor, which decreases the absorption of fats from the gut by approximately $30 \% .^{31,32}$

Another type of antiobesity drugs are "appetite suppressant" agents that act in the central nervous system. This type includes lorcaserin, naltrexone/bupropion, and phentermine/ topiramate. ${ }^{32}$ Liraglutide is a GLP-1 receptor agonist, affecting both the gastrointestinal tract and the brain. ${ }^{33}$

Lorcaserin is a selective agonist of the 5-HT2C receptor. A 5-HT2C receptor agonist regulates appetite modulating the serotonin system. In fact, this receptor possesses anorexigenic properties and is able to promote weight loss. Activation of 5 -HT2C receptors in the hypothalamus promotes satiety by stimulating POMC production. Furthermore, the use of lorcaserin is associated with significant weight loss and improved glycemic control in patients with type 2 diabetes mellitus. ${ }^{31-33}$

Naltrexone/bupropion and phentermine/topiramate are drug combinations designed to target the dopamine reward system. Both bupropion and naltrexone alone produce modest weight loss, while the combination exerts a synergistic effect, ending in a more profound weight reduction. On the other side, phentermine and topiramate are prescription drugs that have been used in the treatment of obesity as monotherapy for years (phentermine) or for epilepsy (topiramate). Taken together, these two substances induce weight loss by suppressing appetite, also making patients feel full, and thus more compliant with diet plans. ${ }^{32,33}$

Liraglutide as a GLP-1 receptor agonist and exerts its effect on food intake and weight through actions in the stomach and gut, but also through direct and indirect effects on the central nervous system, but the exact routes of action are largely unknown. GLP-1 affects the gastrointestinal system and delays absorption of food. This is caused by several means, including decreased gastric emptying and acid secretion. Combined, these gastrointestinal effects reduce the meal-related increase in glucose. Prolonged presence of food in the stomach through delayed gastric emptying may 
also reduce energy intake by inducing satiety. Additionally, GLP-1 receptors are present in several areas in the brain. The receptors in the brainstem (area postrema and subfornical organ) are believed to be involved in inducing satiety, regardless of the presence of food in the gastric system. This action therefore provides other means for decreasing energy intake. ${ }^{33,34}$

Since stabile long-term and profound weight reduction as a desired treatment goal is difficult to achieve with currently available antiobesity drugs alone, diet and proper physical activity are still of greatest importance in obesity management. However, both lifestyle change and medical treatment are associated with weight regain in those with poor treatment adherence or after treatment termination. ${ }^{35}$ The only contemporary therapy that provides sustainable weight reduction is bariatric surgery, leading to caloric deprivation, weight loss, and often to regression of other components of the metabolic syndrome like type 2 diabetes, arterial hypertension, and dyslipidemia. However, the mechanisms of action that result in weight loss after bariatric procedures are not completely understood. A significant proportion of the resulting reduction in caloric intake may not be a result of the restrictive and malabsorptive mechanisms and is thought to be mediated by changes in neuroendocrine function, since bariatric surgery alters the gut hormone profile. Bariatric surgery may alter brain activation patterns, the desire to eat, and taste preferences. For instance, postsurgical reductions in ghrelin and earlier and enhanced postprandial elevations of PYY and GLP-1 may promote satiety. On the other hand, there are changes in brain activation following bariatric procedures, like altered brain dopamine signaling, as a part of the reward-related/hedonic mechanisms of human behavior. It remains unclear if those changes are related to comorbid conditions that are able to alter dopamine signaling. However, to develop new pharmacological therapeutic strategies effective against obesity, the physiological mechanism controlling energy homeostasis must be better understood. Only then it will be possible to ensure efficacy, safety, and sustained weight loss. ${ }^{36-38}$

\section{Vaccination as a form of obesity prophylaxis and/or treatment}

The principal idea of antiobesity drugs is to augment anorexigenic and lipolytic signaling, or to block orexigenic and lipogenic mediators. Recent studies have shown that therapeutic vaccines could be a new approach in developing antiobesity medications. A vaccine should provoke an immune response to a specific causal factor for a particular disease. Throughout human history, vaccines helped to eradicate many infectious diseases, but it seems that immunization may be helpful even in the prophylaxis and treatment of noninfectious diseases like certain types of cancer other than cervical and hepatocellular cancer. All vaccines work through the administration of a target substance or antigen, which triggers an immune response resulting in the production of neutralizing antibodies that bind the antigen and remove it from the body. Antigens are typically proteins but may also be a sugar or lipid.

Regarding obesity treatment, therapeutic vaccines could alternatively treat obesity by suppressing appetite-stimulating hormones and/or by blocking nutrient absorption. Regulatory peptides originating from the stomach, gut, pancreas, and adipose tissue were studied as potential targets. ${ }^{39}$ Among others, a variety of potential drugs able to block the action of ghrelin were tested, since it has been demonstrated that ghrelin stimulates food intake leading to adiposity in both rodents and humans.

The first attempts at an anti-ghrelin vaccination strategy consisted of passive antibody transfer. Polyclonal anti-ghrelin antibodies were intracerebroventricularly administered and lead to dose-dependently inhibition of fast-induced feeding and suppressed dark phase food intake in rats. ${ }^{40}$

Ghrelin receptor antagonists were able to improve glucose tolerance, suppress appetite, and promote weight loss in rats, providing further evidence to the idea of ghrelin blocking as a potential treatment target for obesity. In rodents, improvements in glucose homeostasis were detected even after administration of a single oral dose of a GHS-R1a (growth hormone secretagogue receptor type 1a, ghrelin receptor type 1a) antagonist. Improved glucose tolerance was attributed to increased insulin secretion. Furthermore, daily oral administration of a GHS-R1a antagonist to obese mice reduced food intake and led to weight loss (up to 15\%) due to selective loss of fat mass. Pair-feeding experiments indicated that reduction in weight was mainly an effect of reduced food intake. The influence of a GHS-R1a antagonist on gastric emptying was also tested. Although gastric emptying was modestly delayed by the GHS-R1a antagonist at the highest dose tested $(10 \mathrm{mg} / \mathrm{kg})$, it did not appear to be necessary for weight loss since lower doses of the tested GHS-R1a antagonist led to weight loss without effects on gastric emptying. The anorexigenic effects of GHS-R1a antagonists in mice corresponded with their brain exposure, consistent with the theory that ghrelin produces central effects on feeding. ${ }^{41}$

In another study, ghrelin receptor antagonist, [D-Lys(3)] GHRP-6, after 7 days of subcutaneous treatment was shown to decrease food intake markedly in ovariectomized mice fed 
both with high-fat and standard diets. It also reduced body weight and blood glucose, insulin, and leptin levels, and increased $\beta$-hydroxybutyrate concentration and uncouplingprotein-1 mRNA in brown adipose tissue. Pair-feeding showed that the effect of [D-Lys(3)]GHRP-6 was primary anorexigenic. ${ }^{42}$ As an additional proof of this concept, inoculation of monoclonal anti-ghrelin antibodies in mice inhibited acute ghrelin-mediated orexigenic effects.

Lu et al generated and characterized high-affinity anti-acyl ghrelin-specific monoclonal antibodies. In vitro, the lead $\mathrm{mAb}$ (33A) was able to bind specifically to acryl-ghrelin. In recombinant receptor cell-based assays, 33A dose-dependently inhibited the ghrelin-mediated calcium signal, with a halfmaximal inhibitory concentration of approximately $3.5 \mathrm{nM}$. In vivo, ghrelin effects on food intake in mice were fully blocked by a single injection of 33A. In a 4-week extension of the study, these antibodies were shown to effectively bind to endogenous acryl-ghrelin; however, long-term administration of 33A did not affect food intake or body weight gain in this mouse model. The lack of long-term effects on body weight control by $33 \mathrm{~A}$ suggested that compensatory mechanisms could modulate the energy balance regulation. ${ }^{43}$

To identify the possibility of its use as a vaccine, synthetic ghrelin analogs were conjugated to haptens. So, the first vaccine used ghrelin immunoconjugates in order to induce an autoimmune response to this endogenous peptide. It was developed in 2006 and was tested in rats. The investigators prepared three active vaccines based on the 28 -amino acid residue sequence of ghrelin. All three were used to immunize adult male Wistar rats. Synthetic ghrelin analogs were prepared that spanned residues 1-10 (ghrelin [1-10] Ser-3[butanoyl] hapten), 13-28 (ghrelin [13-28] hapten), and 1-28 (ghrelin [1-28] Ser-3[butanoyl] hapten), and included $n$-butanoyl esters at Ser- 3 . The first and third group showed greater and more selective plasma binding capacity for the active Ser-3-(n-octanoyl) form of ghrelin as compared to the second group or keyhole limpet hemocyanin-vaccinated controls. These animals gained less body weight, with sparing of lean mass and favored reduction of body fat, which was related to reduced circulating leptin levels. Furthermore, the ratio of brain/serum ghrelin levels was also lower in rats with strong anti-ghrelin immune responses, possibly consistent with a reduced passage of circulating ghrelin to the central compartment. Effects were not attributable to nonspecific inflammatory responses. ${ }^{44}$

In a further study, pigs were actively immunized against ghrelin. For this study, ghrelin (1-10) was conjugated to bovine serum albumin and emulsified in Freund's incom- plete adjuvant and diethylaminoethyl-dextran. Primary immunization was given at 19 weeks of age, with booster immunizations given 20 days and 40 days after primary immunization. Body weight and plasma ghrelin concentration were measured weekly beginning at 19 weeks of age, and feed intake was measured daily. Fourteen days after primary immunization, the ratio of bound (125)I-ghrelin in plasma from immunized pigs was significantly increased compared to control animals. Voluntary feed intake was decreased more than $15 \%$ in animals that were actively immunized against ghrelin compared to controls. However, weight loss did not reach the threshold of statistical significance, although immunized pigs weighed approximately $10 \%$ less than animals in the control group. The authors concluded that immunization against ghrelin caused mild anorexia, and that this procedure could potentially be used as a treatment to control caloric intake and obesity. ${ }^{45}$ Limitations of these vaccines were the compulsory use of adjuvants, which may be associated with undesirable effects in humans like inflammatory responses.

Another approach to develop a ghrelin vaccine was to use a noninfectious viral vector carrying ghrelin. ${ }^{46}$ In the recent past, virus-like particles have been used in order to induce the production of specific antibodies against endogenous molecules in several recombinant vaccines designed for chronic noncommunicable diseases. Induced specific autoantibodies were meant to neutralize disease-related proteins offering vaccines to effectively and affordably treat such diseases, like the anti-angiotensin vaccine developed for arterial hypertension treatment. ${ }^{47}$

Following this concept, an anti-ghrelin vaccine was designed to provoke an autoimmune response and to develop antibodies, using a chemical conjugate of active ghrelin with protein tubules of NS1 of the bluetongue virus. The main goal was to develop a safe and effective anti-ghrelin vaccine for obesity. In a randomized study, male adult C57BL/6 mice, with normal weight and with diet-induced obesity, were divided into six weight matched groups, and each group received three intraperitoneal injections with 2-week intervals, containing $75 \mu \mathrm{g}$ of ghrelin-NS1 immunoconjugate, $75 \mu \mathrm{g}$ of NS1, and phosphate buffer solution. Immunized animals presented increased titers of anti-ghrelin antibodies, while their cumulative food intake significantly decreased and energy expenditure was significantly enhanced, although there were no significant changes in body weight. Vaccinated mice also displayed significant decrease of the NPY expression in the basal hypothalamus reflecting a decrease in central orexigenic signals, and showing that the anti-ghrelin vaccine acts even through mechanisms involving 
the brain. This study suggested that this anti-ghrelin vaccine had a positive impact on energy homeostasis and could be an additional therapeutical tool to be used with diet and exercise for obesity treatment. However, despite the fact that immunized animals presented with increased titers of anti-ghrelin antibodies, and their cumulative food intake was significantly decreased and energy expenditure significantly enhanced, there were no significant changes in body weight. ${ }^{46}$

In humans, immunization against ghrelin had entered Phase I and IIa trials as an antiobesity therapy. The results in human studies were not so optimistic. In 2006 there was a randomized, double-blind, and placebo-controlled trial with 87 obese patients aged $18-55$ years with a body mass index between 30 and 35. Participants received four injections of $300 \mu \mathrm{g}$ of the vaccine or placebo at weeks $0,4,8$, and 16 . All study participants were professionally counselled in order to achieve changes in eating habits and improve physical activity. At the end of the study, there was no additional weight loss achieved in comparison to the control group, despite a strong response in ghrelin autoantibodies. The median weight loss was $3.6 \mathrm{~kg}$ after a 6-month period in both groups, but the treatment was safe and well tolerated. ${ }^{48,49}$

On the other hand, a different study showed that IgG anti-ghrelin autoantibodies are able to protect ghrelin from degradation, and autoimmune response might be involved in enhancing ghrelin orexigenic effects. However, the same study also showed that not all obese subjects showed an increased affinity of IgG for ghrelin, suggesting that such an increase is probably not the result of the obese phenotype or at least not its obligatory result. The cause of humans producing ghrelin-reactive $\mathrm{IgG}$ is presently unknown. ${ }^{50} \mathrm{It}$ is tempting to speculate that ghrelin-like antigens are expressed by some kind of gut bacteria, ie, Enterococcus faecalis, indicating that some microbial factors are involved in an exaggerated ghrelin-reactive $\mathrm{IgG}$ production. ${ }^{51}$

\section{Conclusion}

In summary, the anti-ghrelin vaccine decreases food intake, decreases hypothalamic orexigenic signals, and increases energy expenditure, at least in rodents and pigs. Ghrelin has been tested as a potential target to treat obesity by developing therapeutic vaccines, but the development of vaccines to treat these conditions is insufficient at present. Several studies have demonstrated the "proof of concept", but more intensive studies are required to develop prophylactic and therapeutic vaccines to prevent and/or cure obesity. However, caution is necessary due to several findings indicating that some sort of anti-ghrelin antibodies could protect ghrelin from degradation. Therefore, therapeutic vaccines using anti-ghrelin antibodies are still not available as an alternative treatment tool to be used with diet and exercise to treat obesity.

\section{Disclosure}

The authors report no conflicts of interest in this work.

\section{References}

1. Neel JV. Diabetes mellitus: a "thrifty" genotype rendered detrimental by "progress"? Am J Hum Genet. 1962;14:353-362.

2. O'Dea K. Overview of the thrifty genotype hypothesis. Asia Pac J Clin Nutr. 1995;4:339-340.

3. O'rourke RW. Metabolic thrift and the genetic basis of human obesity. Ann Surg. 2014;259(4):642-648.

4. Berbesque JC, Marlowe FW, Shaw P, Thompson P. Hunter-gatherers have less famine than agriculturalists. Biol Lett. 2014;10(1):20130853.

5. Speakman JR. Evolutionary perspectives on the obesity epidemic: adaptive, maladaptive, and neutral viewpoints. Annu Rev Nutr. 2013;33: 289-317.

6. Ng M, Fleming T, Robinson M, et al. Global, regional, and national prevalence of overweight and obesity in children and adults during 1980-2013: a systematic analysis for the Global Burden of Disease Study 2013. Lancet. 2014;384(9945):766-781.

7. Wang Y, Beydoun MA. The obesity epidemic in the United States gender, age, socioeconomic, racial/ethnic, and geographic characteristics: a systematic review and meta-regression analysis. Epidemiol Rev. 2007;29:6-28.

8. Obesity and overweight fact sheet $\mathrm{N}^{\circ} 311$ [webpage on the Internet] Geneva: World Health Organization [updated Jan 2015; cited Jan 2015] Available from: http://www.who.int/mediacentre/factsheets/fs311/en/. Accessed January 3, 2015.

9. Zjacic-Rotkvic V. Endocrinology and immunology of obesity, obesity vaccines. Curr Clin Pharmacol. 2013;8(3):232-237.

10. Skolnik NS, Ryan DH. Pathophysiology, epidemiology, and assessment of obesity in adults. J Fam Pract. 2014;63(7):S3-S10.

11. Mullur R, Liu YY, Brent GA. Thyroid hormone regulation of metabolism. Physiol Rev. 2014;94(2):355-382.

12. Kleinridders A, Ferris HA, Cai W, Kahn CR. Insulin action in brain regulates systemic metabolism and brain function. Diabetes. 2014; 63(7):2232-2243.

13. Izadi V, Saraf-Bank S, Azadbakht L. Dietary intakes and leptin concentrations. ARYA Atheroscler. 2014;10(5):266-272.

14. Blüher M. Adipokines - removing road blocks to obesity and diabetes therapy. Mol Metab. 2014;3(3):230-240.

15. Kojima M, Kangawa K. Ghrelin: structure and function. Physiol Rev. 2005;85(2):495-522.

16. Holtmann G, Talley NJ. The stomach-brain axis. Best Pract Res Clin Gastroenterol. 2014;28(6):967-979.

17. Patterson M, Bloom SR, Gardiner JV. Ghrelin and appetite control in humans - potential application in the treatment of obesity. Peptides. 2011;32(11):2290-2294.

18. Sobrino Crespo C, Perianes Cachero A, Puebla Jiménez L, Barrios V, Arilla Ferreiro E. Peptides and food intake. Front Endocrinol (Lausanne). 2014;5:58.

19. Simpson K, Parker J, Plumer J, Bloom S. CCK, PYY and PP: the control of energy balance. Handb Exp Pharmacol. 2012;(209):209-230.

20. Buhmann H, le Roux CW, Bueter M. The gut-brain axis in obesity. Best Pract Res Clin Gastroenterol. 2014;28(4):559-571.

21. Pocai A. Unraveling oxyntomodulin, GLP1's enigmatic brother. J Endocrinol. 2012;215(3):335-346.

22. Price SL, Bloom SR. Protein PYY and its role in metabolism. Front Horm Res. 2014;42:147-154. 
23. Roth JD. Amylin and the regulation of appetite and adiposity: recent advances in receptor signaling, neurobiology and pharmacology. Curr Opin Endocrinol Diabetes Obes. 2013;20(1):8-13.

24. Filippi BM, Abraham MA, Yue JT, Lam TK. Insulin and glucagon signaling in the central nervous system. Rev Endocr Metab Disord. 2013;14(4):365-375.

25. Wynne K, Stanley S, McGowan B, Bloom S. Appetite control. J Endocrinol. 2005;184(2):291-318.

26. Dhillo WS. Appetite regulation: an overview. Thyroid. 2007;17(5): 433-445.

27. Suzuki K, Jayasena CN, Bloom SR. Obesity and appetite control. Exp Diabetes Res. 2012;2012:824305.

28. Kushner RF, Ryan DH. Assessment and lifestyle management of patients with obesity: clinical recommendations from systematic reviews. JAMA. 2014;312(9):943-952.

29. Hainer V, Toplak H, Mitrakou A. Treatment modalities of obesity: what fits whom? Diabetes Care. 2008;31 Supp1 2:S269-S277.

30. Jauch-Chara K, Oltmanns KM. Obesity - a neuropsychological disease? Systematic review and neuropsychological model. Prog Neurobiol. 2014;114:84-101.

31. Joo JK, Lee KS. Pharmacotherapy for obesity. J Menopausal Med. 2014;20(3):90-96

32. Zhang Y, Liu J, Yao J, et al. Obesity: Pathophysiology and Intervention. Nutrients. 2014;6(11):5153-5183.

33. Hainer V. Overview of new antiobesity drugs. Expert Opin Pharmacother. 2014;15(14):1975-1978.

34. Nanning S, Pucci A, Finer N. Pharmacotherapy for obesity: novel agents and paradigms. Ther Adv Chronic Dis. 2014;5(3):135-148.

35. Soeliman FA, Azadbakht L. Weight loss maintenance: A review on dietary related strategies. J Res Med Sci. 2014;19(3):268-275.

36. Lo Menzo E, Szomstein S, Rosenthal RJ. Changing Trends in Bariatric Surgery. Scand J Surg. 2015;104(1):18-23.

37. Holst JJ. Enteroendocrine secretion of gut hormones in diabetes, obesity and after bariatric surgery. Curr Opin Pharmacol. 2013;13(6): 983-988.

38. Manning S, Pucci A, Batterham RL. GLP-1: a mediator of the beneficial metabolic effects of bariatric surgery? Physiology (Bethesda). 2015; 30(1):50-62.

39. Na HN, Kim H, Nam JH. Prophylactic and therapeutic vaccines for obesity. Clin Exp Vaccine Res. 2014;3(1):37-41.
40. Nakazato M, Murakami N, Date Y, et al. A role for ghrelin in the central regulation of feeding. Nature. 2001;409(6817):194-198.

41. Esler WP, Rudolph J, Claus TH, et al. Small-molecule ghrelin receptor antagonists improve glucose tolerance, suppress appetite, and promote weight loss. Endocrinology. 2007;148(11):5175-5185.

42. Maletínská L, Matyšková R, Maixnerová J, et al. The Peptidic GHS-R antagonist [D-Lys(3)]GHRP-6 markedly improves adiposity and related metabolic abnormalities in a mouse model of postmenopausal obesity. Mol Cell Endocrinol. 2011;343(1-2):55-62.

43. Lu SC, Xu J, Chinookoswong N, et al. An acyl-ghrelin-specific neutralizing antibody inhibits the acute ghrelin-mediated orexigenic effects in mice. Mol Pharmacol. 2009;75(4):901-907.

44. Zorrilla EP, Iwasaki S, Moss JA, et al. Vaccination against weight gain. Proc Natl Acad Sci U S A. 2006;103(35):13226-13231.

45. Vizcarra JA, Kirby JD, Kim SK, Galyean ML. Active immunization against ghrelin decreases weight gain and alters plasma concentrations of growth hormone in growing pigs. Domest Anim Endocrinol. 2007;33(2):176-189.

46. Andrade S, Pinho F, Ribeiro AM, et al. Immunization against active ghrelin using virus-like particles for obesity treatment. Curr Pharm Des. 2013;19(36):6551-6558.

47. Morais T, Andrade S, Pereira SS, Monteiro MP. Vaccines for metabolic diseases: current perspectives. Vaccine (Auckl). 2014;4:55-72.

48. Colon-Gonzalez F, Kim GW, Lin JE, Valentino MA, Waldman SA. Obesity pharmacotherapy: what is next? Mol Aspects Med. 2013;34(1):71-83.

49. Phase I/IIa clinical trial with obese individuals shows no effect of CYT009-GhrQb on weight loss [webpage on the Internet]. Schlieren: Cytos Biotechnology AG; 2006 [updated November 7, 2006; cited January 3, 2015]. Available from: http://www.cytos.ch/news/180/15/ Phase-I-IIa-clinical-trial-with-obese-individuals-shows-no-effect-ofCYT009-GhrQb-on-weight-loss.html. Accessed January 3, 2015.

50. Takagi K, Legrand R, Asakawa A, et al. Anti-ghrelin immunoglobulins modulate ghrelin stability and its orexigenic effect in obese mice and humans. Nat Commun. 2013;4:2685.

51. Fetissov SO, Hamze Sinno M, Coëffier M, et al. Autoantibodies against appetite-regulating peptide hormones and neuropeptides: putative modulation by gut microflora. Nutrition. 2008;24(4):348-359.
ImmunoTargets and Therapy

\section{Publish your work in this journal}

ImmunoTargets and Therapy is an international, peer-reviewed open access journal focusing on the immunological basis of diseases, potential targets for immune based therapy and treatment protocols employed to improve patient management. Basic immunology and physiology of the immune system in health, and disease will be also covered. In addition, the journal will focus on the impact of manage-

\section{Dovepress}

ment programs and new therapeutic agents and protocols on patient perspective such as quality of life, adherence and satisfaction. The manuscript management system is completely online and includes a very quick and fair peer-review system, which is all easy to use. Visit http://www.dovepress.com/testimonials.php to read real quotes from published authors. 